\title{
Complejidad sintáctica en narraciones de niños con desarrollo típico, trastorno específico del lenguaje y discapacidad intelectual
}

\author{
Pedro Alfaro-Faccio - pedro.alfaro@pucv.cl \\ Nina Crespo Allende - nina.crespo@pucv.cl \\ Carola Alvarado Barra - carola_alvarado@mi.cl \\ Pontificia Universidad Católica de Valparaíso
}

Rebut / Received: 5-6-15

Acceptat / Accepted: 29-2-16

\begin{abstract}
Resum. Complexitat sintàctica en narracions de nens amb desenvolupament típic, trastorn específic del llenguatge i discapacitat intel-lectual ${ }^{1}$. El desenvolupament tardà del llenguatge es defineix com la complexització de la llengua amb posterioritat a l'adquisició inicial i durant els primers anys d'escolarització. En aquest context, aquest treball cerca descriure com succeeix aquest procés en nens de tres poblacions diferents: desenvolupament típic (DT), trastorn específic del llenguatge (TEL) i discapacitat intel-lectual (DI). Per això, s'analitzen, des d'una perspectiva funcional-discursiva, les narracions orals de tres grups de nens xilens. La mostra la constituïren escolars amb DT=41, TEL=38 i DI=17, els quals van recontar una història presentada en format audiovisual. Dels resultats, en destaca que la diferència entre els tres grups no radica en la quantitat de vincles interclausulars utilitzats, sinó en els tipus de relacions interclausulars que prefereixen per a construir les seves narracions a l'interior dels paquets de clàusules.
\end{abstract}

Paraules clau: complexitat sintàctica, narració oral, desenvolupament típic, discapacitat intel.lectual, trastorn específic del llenguatge.

1. Investigación desarrollada en el marco del proyecto Factores lingüísticos y cognitivos que se relacionan con el desarrollo de la complejidad sintáctica oral en los primeros años escolares, FONDECYT 1130420. 


\begin{abstract}
Syntactic complexity in storytelling of children with typical development, specific language impairment, and intellectual disability. Later language development is defined as the increase in language complexity that occurs after the period of initial acquisition and during earlier years of schooling. In this context, this paper aims to describe how this process occurs in three different children populations: typical development (TD), specific language impairment (SLI) and intellectual disability (ID). To do this, the oral narratives of three groups of Chilean children are analyzed from a functional-discourse perspective. The sample consisted of TD=41, $\mathrm{SLI}=38$ and ID=17 school-aged children, who retold a story presented in audiovisual format. The results show that the difference between the three groups is not based in the amount of interclause links used, but in the interclause relation types they prefer to build their stories into clause packages. The results indicate that the difference between the three groups is not based in the amount of interclause links used, but in the interclause relation types they prefer to build their stories into clauses packages.
\end{abstract}

Keywords: syntactic complexity, storytelling, typical development, intellectual disability, specific language impairment.

\title{
1. Introducción
}

Durante los primeros años de escolaridad, el lenguaje oral sufre cambios cuantitativos y cualitativos; dentro de ellos emerge el fenómeno de la complejización de la sintaxis con que los niños elaboran sus discursos. No obstante, estos cambios no ocurren de igual modo en todas las poblaciones de escolares. En el caso de Chile, conocer y valorar estas diferencias se torna crucial, dado el marco de inclusión que ha propuesto desde el Ministerio de Educación (MINEDUC, 2009). En el marco del proyecto FONDECYT 1130420 , la pregunta que guía este trabajo es cómo ocurre la complejidad de la sintaxis en las narraciones orales producidas por niños de dos poblaciones con necesidades educativas especiales (trastorno específico de lenguaje y discapacidad intelectual) respecto de niños sin discapacidad. Así el objetivo de este consiste en describir y comparar dicha complejidad en el discurso de estas poblaciones y, con ello, contribuir su caracterización lingüística.

\section{Complejidad sintáctica}

La complejidad sintáctica ha sido definida de diversos modos, de acuerdo con las líneas de investigación con las que se ha abordado el fenómeno. En efecto, es posible distinguir al menos tres perspectivas coexistentes: estructural, generativa y funcionaldiscursiva. Desde cada de uno de ellas ha operacionalizado la complejidad de acuerdo 
al modo en que se concibe el lenguaje (Crespo et al. 2011). Así, autores estructuralistas han entendido la complejidad como la presencia un mayor número de ciertas estructuras morfosintácticas por sobre otras (Brown 1973, Gili Gaya 1972); los generativistas la han definido como la aplicación de una mayor cantidad de reglas transformaciones sobre una unidad sintáctica mínima o kernel (Hunt 1965, Véliz 1988); y los funcionalistas la han conceptualizado como el tipo de relación que se establece entre las cláusulas con fines comunicativos (Berman y Slobin 1994, Nir y Berman 2010).

En términos empíricos, los dos primeros enfoques han sido utilizados para dar cuenta cuantitativamente de la complejidad sintáctica en forma longitudinal, a través de diferentes edades y/o el avance en el sistema escolar de los sujetos (Hunt 1965, 1970, Véliz 1988 y Rodríguez 2008). El enfoque funcional-discursivo, por su parte, ofrece una alternativa que permite analizar este fenómeno tanto cuantitativa como cualitativamente (Berman y Slobin 1994, Katzenberger 2003 y Nir y Berman 2010).

Desde esta última perspectiva, se plantea que las cláusulas se distribuyen y organizan dentro de los textos en unidades intermedias, guiadas por criterios sintácticos, temáticos y discursivos, llamadas paquetes clausulares (PC) (Katzenberger 2003). El modo en que se relacionan las cláusulas al interior de cada PC genera una Arquitectura Interclausular, la cual, a su vez, determina qué tan complejo es un paquete respecto de otro. La complejidad, entonces, depende de los tipos de vínculos presentes dentro de los PCs, pues algunas relaciones interclausulares serían más complejas que otras. En esta línea, Nir y Berman (2010) plantean, respecto del desarrollo de la complejidad, que dentro de un PC pueden darse cinco tipos de relaciones entre las cláusulas, con grados crecientes de complejidad, como ejemplifica la Tabla 1:

TABLA I. EJEMPLO DE RELACIONES INTERCLAUSUlARES

\begin{tabular}{|l|l|l|}
\hline $\begin{array}{l}\text { Tipo de } \\
\text { relación }\end{array}$ & Definición operativa & Ejemplo \\
\hline Isotaxis & $\begin{array}{l}\text { cláusulas aisladas autónomas } \\
\text { o cláusula principal }\end{array}$ & $\begin{array}{l}\text { "una mariposa voló } \\
\text { por las nubes" }\end{array}$ \\
\hline $\begin{array}{l}\text { Parataxis } \\
\text { Simétrica }\end{array}$ & $\begin{array}{l}\text { cláusulas yuxtapuestas y coordinadas, } \\
\text { relacionadas por cohesión semántica y } \\
\text { temática, independientes gramaticalmente }\end{array}$ & $\begin{array}{l}\text { "él iba a buscar una red y } \\
\text { quería atrapar la mariposa" }\end{array}$ \\
\hline $\begin{array}{l}\text { Parataxis } \\
\text { Asimétrica }\end{array}$ & $\begin{array}{l}\text { cláusulas yuxtapuestas y coordinadas, } \\
\text { relacionadas por cohesión } \\
\text { morfosintáctica, semántica y temática, } \\
\text { dependientes gramaticalmente }\end{array}$ & $\begin{array}{l}\text { "y volaron muy alto las } \\
\text { mariposas y acá las atrapó” }\end{array}$ \\
\hline
\end{tabular}




\begin{tabular}{|l|l|l|}
\hline Hipotaxis & $\begin{array}{l}\text { cláusulas subordinadas que } \\
\text { cumplen funciones sintácticas } \\
\text { dentro de otra subordinante }\end{array}$ & $\begin{array}{l}\text { "las mariposas veían } \\
\text { que alguien estaba } \\
\text { viendo del árbol” }\end{array}$ \\
\hline Endotaxis & $\begin{array}{l}\text { cláusulas parentéticas y con } \\
\text { incrustación central }\end{array}$ & $\begin{array}{l}\text { "las mariposas que estaban } \\
\text { volando escaparon" }\end{array}$ \\
\hline
\end{tabular}

Este último enfoque será el que se utilice en esta investigación, dado que permitirá caracterizar la sintaxis de los grupos en estudio y, a la vez, establecer comparaciones entre ellos.

\section{Trastorno específico del lenguaje}

El trastorno específico del lenguaje (TEL) corresponde a un desorden del desarrollo, altamente heredable (Bishop 2009, Villanueva et al. 2011 y Badcock et al. 2011), que alcanza una prevalencia estimada en Chile de un $4 \%$ de los niños entre 3 y 7 años, con una mayor frecuencia en varones (De Barbieri et al. 1999). Este trastorno se caracteriza por una "anormal adquisición de la comprensión o expresión del lenguaje hablado o escrito, en ausencia de retraso mental, problemas sensoriales, neurológicos, emocionales, conductuales severos y deprivación sociocultural" (American Speech-Language-Hearing Association (ASHA) 1980, p. 317). Debido a que las alteraciones observadas en los niños con TEL pueden implicar uno, alguno o todos los niveles del lenguaje -fonología, morfología, sintaxis, semántica, pragmática-, se afirma que no es una condición homogénea (ASHA 1980). Carballo (2012) señala que en la población con TEL existirían diferentes perfiles de déficits en el sistema lingüístico y con distintos grados de severidad. Pese a la tipificación de "específicos", se ha sostenido que las alteraciones verbales coexisten con un amplio rango de dificultades cognitivas no lingüísticas (Van der Lely 2005; Carballo 2012; Mendoza 2012) entre las que se contemplan déficits atencionales (Ebert y Kohnert 2011), perceptivos (Ziegler et al. 2011), motores (Iverson y Braddock 2011) o de memoria (Archibald et al. 2011). Además, se ha afirmado que los niños diagnosticados con TEL presentarían riesgo de manifestar problemas conductuales, emocionales, sociales y de aprendizaje asociados (López-Rubio et al. 2010, St Clair et al. 2011 y Carballo 2012). En términos evolutivos, se plantea que, a pesar de que las terapias parecen ser efectivas, los TEL se mantienen en el tiempo, incluso hasta la adolescencia y adultez y se manifiestan según las exigencias que enfrenta el niño en su ambiente social y escolar (St. Clair et al. 2011 y Carballo 2012).

Diferentes estudios han confirmado que la sintomatología nuclear del TEL -en comparación con los niños con desarrollo típico- radica en las dificultades morfosintácticas: inconsistencia de la concordancia, uso incorrecto de los nexos, utilización anómala de los morfemas gramaticales y palabras funcionales (Leonard 2000 y Contreras y Mendoza 2011). Respecto de la sintaxis, los niños con TEL utilizan menos cláusulas (Marinellie 2004) y presentan menos las oraciones complejas y palabras más cortas (Scott y Windsor 2000; 
McConnell 2010), las que contienen, a su vez, menos gramaticalidad (Shuele y Dykes 2005 y Scott y Windsor 2000). No obstante, no todos los estudios presentan las mismas conclusiones. Blake et al. (2004), por ejemplo, no encontraron diferencias en la longitud de oraciones, en el uso de morfemas ni en la complejidad sintáctica, al comparar niños con TEL, con desarrollo típico y sin TEL homologados en su desempeño al TEL. En el caso del español de Chile, se ha observado que los niños con TEL se distinguen de aquellos con desarrollo típico en el número de oraciones gramaticales y agramaticales y en el número de oraciones simples y subordinadas sustantivas (Coloma 2013). Asimismo, se ha demostrado la existencia de una correlación significativa entre la sintaxis compleja y el discurso narrativo (Pavez et al. 2001, Pavez y Coloma 2005).

Como demuestra la literatura especializada, los rasgos morfosintácticos del TEL aún son materia de discusión.

\section{Discapacidad intelectual}

La discapacidad intelectual (DI) es un constructo utilizado para describir las dificultades de las personas para alcanzar aprendizajes esperados y funcionar normalmente en la vida cotidiana. Se caracteriza por limitaciones significativas tanto en el funcionamiento intelectual -razonamiento, planificación, resolución de problemas, pensamiento abstracto, comprensión de ideas complejas, aprendizaje rápido y aprendizaje mediante la experiencia (Gottfredson 1997) - como en el comportamiento adaptativo -habilidades conceptuales, sociales y de adaptación práctica, originadas antes de los 18 años (Schalock 2009, Asociación Americana de Psiquiatría 2013).

Respecto del funcionamiento intelectual, para un diagnóstico de DI se consideran, al menos, dos desviaciones estándar por debajo de la media, atendiendo al error estándar de la media para el instrumento de evaluación específico utilizado y los puntos fuertes y limitaciones del instrumento (Schalock 2009). Con esta dimensión se establecen los principales niveles psicométricos del DI como: leve [CI=49-70], moderado [CI=35-49], severo [CI=25-34] y profundo [CI=0-24] (Rondal y Buckley 2003). No obstante, es necesario aclarar que en la actualidad la Asociación Americana de Psiquiatría opta por una evaluación por dominio conceptual, social y práctico para establecer la gravedad del trastorno. El comportamiento adaptativo, por su parte, es entendido como un conjunto de habilidades prácticas, sociales y conceptuales aprendidas, útiles para funcionar en la vida cotidiana (Verdugo 2003). En este marco, las limitaciones significativas en el comportamiento adaptativo se definen como: (a) un rendimiento que sea al menos dos desviaciones estándar por debajo de la media de cada uno de los siguientes tres tipos de comportamiento adaptativo: conceptual, social o práctico; o (b) una puntuación general en una medida estandarizada de habilidades conceptuales, sociales y prácticas (Luckasson et al. 2002).

En cuanto al lenguaje, se ha descrito que los problemas son menores en los niños con DI leve si se les compara con nińos con DI moderada. Sin embargo, en ambos casos se manifiestan 
dificultades de orden discursivo, pragmático, morfosintáctico y léxico. Las personas con niveles severo y profundo, por su parte, presentan muy poco lenguaje de forma espontánea (Rondal y Buckley 2003). Respecto del componente específicamente morfosintáctico, la mayoría de las investigaciones suelen basarse en la producción oral y escrita, desligando el nivel sintáctico de los demás componentes del lenguaje o aspectos relacionados con su uso, por lo que se disponen de escasos datos respecto de la complejidad sintáctica de la oralidad (Garayzábal 2006). Entre los autores que han investigado este fenómeno se encuentran Diez-Itza y Miranda (2007) quienes han señalado que los niños con Síndrome de Down emiten discursos en los cuales las categorías gramaticales más afectadas son los artículos y algunas conjunciones. En otro estudio, Price et al. (2008) comparan niños con síndrome de X frágil y niños con Síndrome de Down y con desarrollo típico. Los resultados de este estudio revelaron que, si bien los dos grupos con DI presentan un retraso sintáctico respecto de sus pares con desarrollo típico, ambos tienen perfiles lingüísticos diferentes.

Esta escasa y dispar información, junto a la enorme diversidad lingüística entre los sujetos con DI, hace urgente investigar el desarrollo sintáctico de los sujetos, intentando encontrar algunos aspectos que pudieran caracterizarla con más precisión a esta población.

\section{Metodología}

Este es un estudio descriptivo-comparativo entre tres grupos: TEL, DI y desarrollo típico (DT). Se recogieron datos en tres muestras con la finalidad de observar su comportamiento y, a partir de allí, analizar sus similitudes y/o diferencias. Este estudio fue validado por el comité de Bioética de la Pontificia Universidad Católica de Valparaíso, Chile (Receptado con fecha 08-05-2013).

\subsection{Participantes}

La muestra estuvo constituida por 96 sujetos, quienes oscilaban entre los 5 y 10 años. Como criterio de unificación se consideró que todos cursaran el último nivel de preescolar en establecimientos escolares regulares y especiales a los que asistían, debido a que las edades de los sujetos del grupo DI es altamente variable y no se corresponden con los años de escolarización. En la Tabla 2, se presenta la distribución de la muestra.

TABla 2. Distribución de la muestra

\begin{tabular}{|l|l|l|l|l|l|l|}
\hline \multirow{2}{*}{} & \multicolumn{3}{|l|}{ Edad } & \multicolumn{3}{l|}{ N $^{\circ}$ de participantes } \\
\cline { 2 - 7 } & mín. & máx. & media & masc. & fem. & subtotal \\
\hline Grupo DT & $4 a 11 \mathrm{~m}$ & 5a 8m & $5 \mathrm{a} 3 \mathrm{~m}$ & 23 & 18 & 41 \\
\hline
\end{tabular}




\begin{tabular}{|l|l|l|l|l|l|l|}
\hline Grupo TEL & 5a $1 \mathrm{~m}$ & 5a $11 \mathrm{~m}$ & $5 \mathrm{a} 6 \mathrm{~m}$ & 13 & 25 & 38 \\
\hline Grupo DI & 5a $5 \mathrm{~m}$ & 9a $3 \mathrm{~m}$ & $7 \mathrm{a} 4 \mathrm{~m}$ & 13 & 4 & 17 \\
\hline TOTAL
\end{tabular}

Los sujetos del grupo TEL asistían, ya sea, a una escuela especial, una especial de lenguaje o a un proyecto de integración en establecimientos regulares de las ciudades de Valparaíso y Viña del Mar. Siguiendo los criterios para el diagnóstico de los TEL, propuestos en el decreto $n^{\circ} 170$, basados en el DMS-IV, los niños de este grupo manifestaban alteraciones del lenguaje en presencia de un desempeńo cognitivo y una audición normal, evidenciables tras la aplicación del STSG-E, STSG-R, TEPROSIF-R, Test de Matrices Coloreadas de Raven (2005) y una evaluación auditiva (audiometría tonal e impedanciometría).

En cuanto al grupo DI, para su selección se consideraron los diagnósticos facilitados por las instituciones a las cuales asistían los niños. Todos los sujetos se encuentran en los rangos de DI leve y moderada, sin síndromes asociados, según escala descrita en el CIE-101, Clasificación Estadística Internacional de Enfermedades y otros, utilizado en el sistema chileno de salud pública (MINEDUC 2009). Este grupo está constituido por casi la mitad de sujetos de los grupos de niños con TEL y DT, debido a que, de un total de 60 niños entrevistados, solo 17 fueron capaces de responder a la tarea narrativa oral de forma efectiva. Todos pertenecían a una escuela especial de la comuna de Santiago.

Los participantes del grupo DT estaban escolarizados en el sistema de educación regular en establecimientos de las ciudades de Viña del Mar y Valparaíso. Todos presentaban un desempeño cognitivo y lingüístico normal, y su escolarización seguía los estándares normales, según el criterio de la educadora a cargo del nivel.

\subsection{Elicitación del discurso}

Para recopilar el corpus de análisis se usó una tarea de recontado audiovisual. Esta consiste en el cuento La mariposa Flopi ${ }^{2}$, estructurado en dos episodios, siguiendo la gramática de las historias de Stein y Glen (1979), Peterson y McCabe (1983), Owens (1995) y Pavez et al. (2008). En cuanto al diseño del apoyo gráfico, las imágenes fueron elaboradas siguiendo la gramática visual de Kress y van Leeuwen (2008).

Respecto de la aplicación, siguiendo a Eissenbeiss (2010) y Reese (2012), se elaboró un protocolo a través del cual el cuento se presentó en forma individual a los

2. Disponible en https://vimeo.com/91330174. Fecha de consulta: 22.09.16. 
niños en una modalidad audiovisual digital y, luego, ellos lo recontaron apoyándose en un texto en formato libro-álbum que contenía las imágenes. Se busco recrear una situación pragmáticamente verosímil de narración de historias infantiles, por lo que la presentación y el recontado del cuento fueron realizados a dos interlocutores distintos.

\subsection{Análisis de la complejidad sintáctica}

A los textos orales narrativos se les aplicó una pauta de complejidad sintáctica (PCS, en adelante), que mide la cantidad y tipo de vínculos interclausulares (VI, en adelante) con los que fue construido el texto, basada en la propuesta de Nir y Berman (2010) y siguiendo a López García (1999) para la gramática del español. Respecto de la cantidad, se consideran los vínculos isotácticos -indicadores de los paquetes clausulares- de cada texto señalando el número de estos que presentan isotaxis principal, los que dan cuenta de un paquete clausular complejizado, compuesto por diversas cláusulas. En cuanto al tipo de vínculo se consideraron fundamentalmente cuatro: parataxis simétrica, parataxis asimétrica, hipotaxis y endotaxis A partir de allí se elaboraron tres perfiles a fin de dar cuenta de los vínculos usados por los nińos de cada una de las tres poblaciones.

\section{Resultados}

Debido a que el mecanismo para incorporar información en los textos son los PC y los vínculos al interior de estos, se analizó el número de paquetes clausulares por cada una de las narraciones y, al mismo tiempo, se determinó cuántos de estos paquetes estaban constituidos por más de una cláusula. La Tabla 3 describe la media, la desviación estándar y el coeficiente de variación de los PC y los PC complejizados.

Tabla 3. Datos descriptivos de PC y PC complejizados

\begin{tabular}{|l|c|c|c|c|c|c|}
\hline \multirow{2}{*}{} & \multicolumn{3}{|c|}{ PC } & \multicolumn{3}{|c|}{ PC complejizado } \\
\cline { 2 - 7 } & $\overline{\mathbf{x}}$ & $\sigma$ & $\mathbf{C . V .}$ & $\overline{\mathbf{x}}$ & $\sigma$ & C.V. \\
\hline Grupo DT & 7,5 & 2,7 & $36 \%$ & 3,6 & 2,0 & $56 \%$ \\
\hline Grupo TEL & 6,0 & 2,0 & $33 \%$ & 2,2 & 1,6 & $73 \%$ \\
\hline Grupo DI & 6,5 & 4,1 & $63 \%$ & 3,2 & 1,9 & $59 \%$ \\
\hline
\end{tabular}


Como se observa en la Tabla 3, tanto en el promedio de PC por narración como en el promedio de PC complejizado, el grupo DT es superior al grupo TEL y DI, lo que indica que sus narraciones contienen más información y una mayor extensión. Este dato parece predecible, dadas las patologías de sujetos TEL y DI. En este sentido, se optó por aplicar la prueba de Kruskall-Wallis para determinar si existen diferencias significativas $(p<0,05)$ entre el comportamiento de los grupos. La Tabla 4 muestra los resultados de dicho análisis.

Tabla 4. Test Kruskall-Wallis

\begin{tabular}{|l|c|c|c|}
\hline & DT - TEL & DT - DI & TEL - DI \\
\hline $\mathbf{N}^{\circ}$ de PC & 0,00278 & 0,2562 & 0,6785 \\
\hline $\mathbf{N}^{\circ}$ de PC complejizado & 0,002488 & 0,5278 & 0,08085 \\
\hline
\end{tabular}

Como muestra la Tabla 4, respecto de promedio de PC por narración, el grupo DT solo se diferencia significativamente del grupo TEL y este último no se diferencia del grupo DI. Lo mismo ocurre respecto del promedio de PC complejizado. Esto indica que los sujetos con TEL incorporan menos información a los núcleos informativos de sus narraciones. Si bien el grupo DI también incluye un menor número de PC y PC complejizados, parece estar entre ambos grupos, al no diferenciarse significativamente de ninguno de estos.

Ahora bien, estos datos señalan la cantidad de información con que se construyen los diversos momentos de las narraciones. Para describir la manera en que se conecta la información al interior de los PC en la Tabla 5 se describe el vínculo interclausular preferido por cada uno de los grupos.

TABla 5. ARQuitectura interclausular POR GRUPO

\begin{tabular}{|l|c|c|c|}
\hline & Grupo DT & Grupo TEL & Grupo DI \\
\hline Parataxis simétrica & $31 \%$ & $35 \%$ & $45 \%$ \\
\hline Parataxis asimétrica & $19 \%$ & $23 \%$ & $29 \%$ \\
\hline Hipotaxis & $51 \%$ & $42 \%$ & $26 \%$ \\
\hline Endotaxis & $0 \%$ & $0 \%$ & $0 \%$ \\
\hline
\end{tabular}


Como muestran los datos, los grupos difieren en cuanto al tipo de relación interclausular que prefieren. Mientras el grupo DT utiliza de forma similar vínculos paratácticos (49\%) e hipotácticos (51\%), los grupos TEL y DI prefieren las relaciones paratácticas (58\% y $74 \%$, respectivamente) por sobre las hipotácticas ( $42 \%$ y $26 \%$, respectivamente). Debido a que los vínculos hipotácticos necesitan mecanismos de concordancia interclausular explícitos para marcar la subordinación (por ejemplo, en las oraciones que expresan deseo, el verbo principal se usa en indicativo y exige una concordancia temporal con verbo subordinado en subjuntivo), consideramos su utilización como indicio de mayor complejidad. En este sentido, si comparamos los grupos se observa un orden creciente de complejidad de las narraciones, que va desde el grupo DI (26\%) al grupo TEL (42\%) y al grupo DT (51\%). No obstante, al realizar análisis inferencial -Kruskall-Wallis- estas diferencias no resultan significativas $(p=0.68)$.

Respecto de la parataxis, en los tres grupos se observa una tendencia a preferir relaciones simétricas -yuxtapuestas y coordinadas- por sobre relaciones asimétricas -en las que hay elementos explícitos de cohesión, que otorgan mayor complejidad a los textos-. Este aspecto, sumado al anterior, denota un menor dominio de elementos que permiten la cohesión morfosintáctica y la integración de oraciones en un mismo PC por parte de los grupos TEL y DI, frente al grupo DT. Presentamos algunos ejemplos de paquetes clausulares construidos por sujetos con DT, TEL y DI, que grafican esta afirmación.

(1) l'sus a 'mi $y a z$ ði 'xe ron 'ke a 're mos i la 'o tra 'ði xo ke 'kwaN do se 'ke e ðor 'mi o la ' $\beta a$ mos a sal ' $\beta a r$ i 'fwe rog ka $\lambda a$ 'i tas 'por la $\beta e N$ 'ta na/ 'sus amigas dijeron: “¿qué haremos?”. Y la otra dijo que cuando se que[d]e dormido, la vamos a salvar. $Y$ fueron calla[d]itas por la ventana'

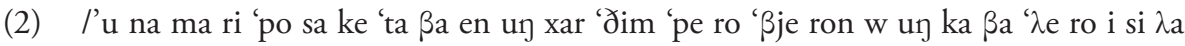
'ma $\beta$ a $\beta$ i' $\gamma o$ tel 'una mariposa que taba en un jardín pero vieron u un caballero y si llamaba bigote'

(3) /se 'po so 'tis te se a řan 'ko/ 'se poso triste... se arrancó'

Como muestran los ejemplos los sujetos del grupo DT (1) utilizan una mayor diversidad de mecanismos que permiten vincular cláusulas - por ejemplo, conjunciones ante completivas, discurso directo-, frente al grupo TEL (2) que solamente hace uso de conjunciones o el grupo DI (3) que solo yuxtapone cláusulas. A nuestro entender, esto otorga, en gran medida, una mayor complejidad a sus narraciones. Cabe señalar que ninguno de los sujetos de los tres grupos produjo estructuras endotácticas, lo que se explica, tal como han señalado Crespo et al. (2011), que este tipo de construcciones serían más usuales en otros tipos de textos y en niños mayores y con más experiencia en el sistema escolar. 


\section{Conclusiones}

Como mostraron los resultados, en lo que se refiere a la extensión en términos de PC y a su complejización, solo hay diferencias en el grupo TEL. Por ello, sería posible aseverar que si bien todos los grupos evidencian una capacidad de construir narraciones deslindando unidades sintácticas, semánticas y discursivas y, a su vez, interrelacionando cláusulas a través de vínculos sintácticos, los sujetos con TEL lo hacen de manera más simple. Esto puede implicar que las tres poblaciones "tienen conciencia" de que para construir un texto auto gestionado deben elaborar unidades temáticas intermedias que contengan unidades sintácticas menores vinculadas entre sí, pero que el grupo TEL no lo hace de manera tal que hay menos información en cada una de dichas partes. Este rasgo de la producción lingüística de los sujetos con TEL podría indicar que no solamente son los niveles fonológico y morfosintáctico los que se ven afectados por esta patología. En esta misma línea, resulta interesante que el DI no se diferencie del grupo DT y sí lo haga del grupo TEL. La descripción de los rasgos del comportamiento lingüísticos de este tipo de sujetos describe alteraciones en todos lo niveles del lenguaje, no obstante, en este caso han narrado con una extensión similar a los niños sin patología. En este sentido, se hace necesario indagar en este aspecto discursivo de la producción lingüística de esta población para corroborar o desestimar estos hallazgos.

Junto a estos resultados, la arquitectura interclausular de los PC también diferencia a los grupos. La selección de tipo de vínculos entre las cláusulas parece indicar una mayor elaboración en las narraciones de DT. En efecto, dado que la hipotaxis se considera más compleja que la parataxis, se puede concluir que la diferencia entre las poblaciones no depende de la cantidad de vínculos que se establecen, sino del tipo de relación que escogen para relacionar la información proposicional. De esta manera, los niños con DT tienden a construir usando más vínculos hipotácticos que los niños de las otras dos poblaciones, que prefieren la parataxis. En el caso del grupo DI las relaciones entre cláusulas son mayoritariamente paratácticas, es decir, construyen PC y los acompañan de cláusulas que los complementan, pero las vinculan en forma simple. El gupo TEL se encuentra a medio camino de estas dos poblaciones.

En suma, estos resultados permiten diferenciar dos aspectos de la producción lingüística de estos sujetos: (a) informatividad de los textos, dada por la cantidad de PC y su complejización, esto es, las partes del texto que explicitan y la cantidad de información que incorporan a dichas partes; y (b) cohesión del contenido dentro de cada PC, esto es, la manera en que se vinculan las oraciones del texto. Con la primera de estas categorías se ha podido establecer una menor complejidad del grupo TEL y con la segunda, una menor complejidad del grupo DI. 


\section{Referencias}

American Speech-Language-Hearing Association (1980). Standards for accreditation by the Education and Training Board. Rockville, MD: American Speech-LanguageHearing Association.

Archibald, L. M. D., Edmunds, A., y Joanisse, M. F. (2011). Specific language or working memory impairments: Are there observable differences? Child Language and Teaching Therapy, 27, 294-312.

Asociación Americana de Psiquiatría (2013). Guía de consulta de los criterios diagnósticos del DSM-5. Arlington, VA: Asociación Americana de Psiquiatría.

Badcock, N. A., Bishop, D. V., Hardiman, M. J., Barry, J. G., y Watkins, K. E. (2011). Co-localisation of abnormal brain structure and function in specific language impairment. Brain Language, 120(3), 310-320.

Berman, R., y Slobin, D. (1994). Relatingevents in narrative: A crosslinguistic developmental study. Hillsdale, NJ: Erlbaum.

Bishop. D. V. M. (2009). Specific language impairment as a language learning disability. Child Language Teaching and Therapy, 25, 63-125.

Blake, J., Myszczyszyn, D., y Jokel, A. (2004). Spontaneous measures of morphosyntax in children with Specific Language Impairment. Applied Psycholinguistics, 25, 29-41.

Brown, R. (1973). A first language: The early stages. London: George Allen \& Unwin.

Carballo, G. (2012) Guía para la evaluación del TEL: Algunas consideraciones. Revista de logopedia, foniatría y audiología, 32, 2, 87-93.

Contreras, C., y Mendoza, E. (2011). El rastreo sintáctico y la planificación de sintagmas: Diferencias entre el trastorno específico del lenguaje y la deprivación sociocultural. Revista de logopedia, foniatría y audiología. 31, 1, 47-56.

Coloma, C. J. (2013). Sintaxis compleja y discurso narrativo en escolares con Trastorno Específico del Lenguaje (TEL) (Tesis doctoral). Universidad de Granada, España.

Crespo, N., Alfaro, P. y Góngora, B. (2011). La medición de la sintaxis: Evolución de un concepto. Onomazein, 24(2), 155-172.

De Barbieri, Z., Maggiolo, M. y Alfaro, S. (1999). Trastornos de la comunicación oral en niños que asisten a control de salud en un consultorio de atención primaria. Revista chilena de pediatría, 70(1), 36-40.

Díez-Itza, E., y Miranda, M. (2007). Perfiles gramaticales específicos en el syndrome de Down. Revista de logopedia, foniatría y audiología, 27(4), 161-172.

Ebert, K. D. y Kohnert, K. (2011) Sustained attention in children with primary language impairment: A meta-analysis syndrome. Journal of Speech Language and Hearing Research. 54(5), 1372-1384.

Eissenbeiss, S. (2010). Production methods in language acquisition research. En E. Blom y S. Unsworth (eds). Experimental methods in language acquisition research (p. 11-34). Amsterdam: John Benjamins Publishing Company. 
Garayzábal, E. (2006). Lingüistica clínica y logopedia. Madrid: Antonio Machado Ediciones.

Gili Gaya, S. (1972). Estudios de lenguaje infantil. Barcelona: Bibliograf.

Gottfredson, L. S. (1997). Why g matters: the complexity of everyday life. Intelligence, 24(1), 1-67.

Hunt, K. (1965). Grammatical structures written at three grade levels. Urbana, IL: National Council of Teachers of English.

Hunt, K. (1970). Syntactic maturity in schoolchildren and adults. Monographs of the Society for Research, 35(1), 1-67.

Iverson, J. M., y Braddock, B. A. (2011). Links between language, gesture, and motor skill in children with language impairment. Journal of Speech Language and Hearing Research, 54, 72-86.

Katzenberger, I. (2003). The development of clause packaging in spoken and written texts. Journal of Pragmatics 36(10), 1921-1948.

Kress, G., y Van Leeuwen, T. (2008). Visual grammar. Londres: Equinox.

Leonard, L. (2000). Specific language impairment across languages. En D. V. M. Bishop y L. B. Leonard (eds.), Speech and language impairments in children: Causes, characteristics, intervention and outcome (p. 115-130). Hove: Psychology Press.

López García, A. (1999). Relaciones paratácticas e hipotácticas. En I. Bosque y V. Demonte (eds.) Gramática descriptiva de la lengua española (p. 3507-3547). Madrid: Editorial Espasa.

López-Rubio, S., Fernández, A., Mendoza, E. y Ramos, C. (2010). Lenguaje y conducta: Determinantes del fracaso académico y social. Una revisión de la literatura. Revista de logopedia, foniatría y audiología, 30, 144-150.

Luckasson, R., Borthwick-Duffy, S., Buntinx, W. H. E., Coulter, D. L., Craig, E. M., Reeve, A., Shalock, R. L., Snell, M. E., Spitalnik, D. M., Spreat, S., y Tassé, M. J. (2002). Mental retardation: Definition, classification, and systems of supports. 10th ed. Washington (DC): American Association on Mental Retardation.

Marinellie, S. A. (2004). Complex syntax used by school-age children with specific language impairment (SLI) in child-adult conversation. Journal of Communication Disorders, 37, 517-533.

McConnell, S. (2010). Sentence complexity in children with autism and specific language impairment (Tesis doctoral). Universidad de Iowa, Estados Unidos.

Mendoza, E. (2012). La investigación actual en el Trastorno Específico del Lenguaje. Revista de logopedia, foniatría y audiología, 32(2), 34-46.

Ministerio de Educación. (2009). Marco curricular. Educación regular. Formación general para Educación Básica y Media, Santiago: MINEDUC.

Nir, B. y Berman, R. (2010). Complex syntax as a window on contrastive rhetoric. Journal of Pragmatics, 42(3), 744-765. 
Owens, R. (1995). Language disorders: A functional approach to assessment and intervention ( $2^{\mathrm{a}}$ ed.). Boston: Allyn and Bacon.

Pavez, M., Coloma, C., y González, P. (2001). Discurso narrativo y desempeño gramatical en niños con Trastorno Específico del Lenguaje. Revista de logopedia, foniatría y audiología, 21(3), 124-130.

Pavez, M., y Coloma, C. (2005) Desarrollo del discurso narrativo en niños con trastorno específico del lenguaje. En Harvey, A. (compiladora) En torno al discurso (p. 149156). Santiago: Ediciones Universidad Católica de Chile.

Pavez, M., Coloma, C., y Maggiolo, M. (2008). El desarrollo narrativo en niños. Una propuesta práctica para la evaluación y la intervención en niños con trastorno del lenguaje. Barcelona: ArsMedica.

Peterson, C., y McCabe, A. (1983). Developmental psycholinguistics: Three ways of looking at a child's narrative. New York: Plenum.

Price, J., Roberts, J., Hennon, E., Berni, M., Anderson, K., y Sideris, J. (2008). Syntactic complexity during conversation of boys with fragile $\mathrm{X}$ síndrome and Down síndrome. Journal of Speech Language and Hearing Research, 51(1), 3-15.

Raven, J. C. (2005). Test de matrices progresivas. Escala coloreada. Cuaderno de Matrices/ Series $A, A B$ y $B$. Buenos Aires: Paidós.

Reese, E., Sparks. A., y Suggate, S. (2012). Assessing children's narratives. En E. Hoff (ed.) Research methods in child language. A practical guide (p.133-148.). Nueva York, NY: Wiley-Balckwell.

Rodríguez, R. B. (2008). La adquisición y el aprendizaje de los modificadores nominales en el discurso escrito de estudiantes de L1 y L2. Revista electrónica de lingüistica aplicada, 7, 45-54.

Rondal, J., y Buckley, S. (2003). Speech and language intervention in Down syndrome. Londres: Whurr Publishers Ltd.

Scott, C., y Windsor, J. (2000). General language performance measures in spoken and written narrative and expository discourse in school-age children with language learning disabilities. Journal of Speech Language and Hearing Research, 43, 324-339.

Schalock, R. (2009). La nueva definición de discapacidad intelectual, apoyos individuales y resultados personales. Revista española sobre discapacidad intelectual, 40(1), 22-30.

St Clair, M. C., Pickles, A., Durkin, K., y Conti-Ramsden, G. (2011). A longitudinal study of behavioral, emotional and social difficulties in individuals with a history of specific language impairment (SLI). Journal of Communication Disorders, 44(2), 186-99.

Schuele, M., y Dykes, J. (2005). Complex syntax acquisition: A longitudinal case study of a child with specific language impairment. Clinical Linguistics \& Phonetics, 19(4), 295-318. 
Stein, N., y Glenn, Ch. (1979). An analysis of story comprehension in elementary school children. En R. Freedle (ed.). Advances in discourse processes: New directions in discourse processing (p. 53-120). Norwood, NJ: Ablex.

Van der Lely, H. K. J. (2005). Domain-specific cognitive systems: Insight from Grammatical specific language impairment. Trends in Cognitive Sciences, 9(2), 53-59. Véliz, M. (1988). Evaluación de la madurez sintáctica en el discurso. Revista de lingüística teórica y aplicada, 26, 105-141.

Verdugo, M.A. (2003). Análisis de la definición de discapacidad intelectual de la Asociación Americana sobre retraso mental de 2002. Siglo Cero, 34(1), 5-19.

Villanueva, P., Newbury, D. F., Jara, L., De Barbieri, Z., Mirza, G., Palomino, H. M., Fernández, M. A., Cazier, J. B., Monaco, A. P., Palomino, H. (2011). Genomewide analysis of genetic susceptibility to language impairment in an isolated Chilean population. European Journal of Human Genetics, 19(6), 687-695.

Ziegler, J., Pech-Georgel, C., George, F., y Lorenzi, C. (2011). Noise on, voicing off: speech perception deficits in children with SLI. Journal of Experimental Child Psychology, 110, 362-372. 